\title{
Antarctic circumpolar transport and the southern mode: a model investigation of interannual to decadal timescales
}

\author{
C. W. Hughes ${ }^{1,2}$, Joanne Williams ${ }^{2}$, A. C. Coward ${ }^{3}$, and B. A. de Cuevas ${ }^{3}$ \\ ${ }^{1}$ School of Environmental Sciences, University of Liverpool, Liverpool, UK \\ ${ }^{2}$ National Oceanography Centre, Liverpool, UK \\ ${ }^{3}$ National Oceanography Centre, Southampton, UK
}

Correspondence to: C. W. Hughes (cwh@liv.ac.uk)

Received: 25 October 2013 - Published in Ocean Sci. Discuss.: 7 November 2013

Revised: 24 January 2014 - Accepted: 23 February 2014 - Published: 10 April 2014

\begin{abstract}
It is well-established that, at periods shorter than a year, variations in Antarctic circumpolar transport are reflected in a barotropic mode, known as the southern mode, in which sea level and bottom pressure varies coherently around Antarctica. Here, we use two multidecadal ocean model runs to investigate the behaviour of the southern mode at timescales on which density changes become important, leading to a baroclinic component to the adjustment. We find that the concept of a southern mode in bottom pressure remains valid, and remains a direct measure of the circumpolar transport, with changes at the northern boundary playing only a small role even on decadal timescales. However, at periods longer than about 5 years, density changes start to play a role, leading to a surface intensification of the vertical profile of the transport. We also find that barotropic currents on the continental slope account for a significant fraction of the variability, and produce surface intensification in the meridional-integral flow. Circumpolar sea level and transport are related at all investigated timescales. However, the role of density variations results in a ratio of sea level change to transport which becomes larger at longer timescales. This means that any long-term transport monitoring strategy based on present measurement systems must involve multiplying the observed quantity by a factor which depends on frequency.
\end{abstract}

\section{Introduction}

The strong Antarctic Circumpolar Current (ACC) represents a stream of approximately $144 \mathrm{~Sv}$, with an uncertainty lying between $8 \mathrm{~Sv}$ and $45 \mathrm{~Sv}$ (Cunningham et al., 2003, $1 \mathrm{~Sv}=1 \mathrm{sverdrup}=10^{6} \mathrm{~m}^{3} \mathrm{~s}^{-1}$ ). It flows in a belt around
Antarctica, through Drake Passage between South America and the Antarctic Peninsula, and also between Antarctica and southern Africa. South of Australia, the ACC is augmented by about $13-15 \mathrm{~Sv}$ of additional flow, which recirculates around Australia in the Indonesian throughflow (Gordon et al., 2010). The current follows a steady path in a band which typically lies between $40^{\circ} \mathrm{S}$ and $60^{\circ} \mathrm{S}$, and at most longitudes lies some way north of the Antarctic continent.

In contrast, early model studies showed that variations in the transport occur in a mode, known as the southern mode, which is strongly steered by topographic contours (more precisely $H / f$ contours, where $H$ is ocean depth and $f$ is the Coriolis parameter) on the Antarctic continental slope, with some extensions to higher latitude along the mid-ocean ridge known as the Pacific Antarctic Rise. This mode cuts across the ACC proper, and results in highly coherent variations of sea level and bottom pressure on all the continental slope surrounding Antarctica (Woodworth et al., 1996; Hughes et al., 1999). The mode is barotropic, is strongly correlated with the atmospheric southern annular mode, and is excited mainly by wind stress in a narrow band close to the Antarctic continental slope (Aoki, 2002; Hughes et al., 2003; Vivier et al., 2005; Weijer and Gille, 2005; Kusahara and Ohshima, 2009; Zika et al., 2013). There is strong observational evidence for the mode in the form of coherent sea level variations from tide gauges and bottom pressure recorders, which are consistent with the predictions of barotropic models (Hughes et al., 1999, 2003; Aoki, 2002; Hughes and Stepanov, 2004; Hibbert et al., 2010). The mode can also be seen in satellite altimetry measurements (Vivier et al., 2005, Hughes and Meredith, 2006), although most of the relevant region is intermittently covered in sea ice, making it difficult to monitor by this method. 
The aim of this paper is to investigate what happens as the timescale is extended from the intra-annual variability, which has been the focus of most of the above investigations, to multidecadal periods. Transport variations cannot be due to barotropic processes at all timescales, not least because the ACC itself, despite penetrating to great depth, is not a barotropic current. On some timescale, presumably, variations must start to take a form comparable with the ACC, with more flow near the surface and a decay to smaller values at depth. This is an issue which was investigated by Olbers and Lettmann (2007) in the context of an idealized ocean model, with only two vertical modes, no eddies, and a smoothed representation of topography. They found a baroclinic timescale of about 16 years, and spectral analysis showed that the role of baroclinic terms was small at periods shorter than about 4 years, rising to play a major role at about a 7-year period. Here we extend their analysis to a more complete ocean model, with realistic geometry and eddies. We will show that the concept of transport determined directly by a southern mode survives intact when interpreted in terms of depth-averaged boundary pressure (bottom pressure on the Antarctic continental slope). However, baroclinic effects start to become important on timescales consistent with the predictions of Olbers and Lettmann (2007), producing a changing relationship between bottom pressure, sea level and transport which allows for the surface intensification required to reflect the geometry of the ACC at long periods. We also find that surface intensification of the meridionally integrated currents can in part be explained by a barotropic mechanism involving currents flowing on the continental slope.

\section{Kinematics}

The relationship between pressure and transport is derived from geostrophic balance, which is generally a good approximation below the Ekman layer on timescales longer than a few days. Consider a constant longitude section, extending from top to bottom of the ocean and from Antarctica to a latitude north of the ACC. If the Coriolis parameter does not change much over the section considered, then the eastward geostrophic volume transport per unit depth across the section is given by

$$
T(z)=\frac{1}{\rho f}\left(p_{\mathrm{S}}-p_{\mathrm{N}}\right),
$$

where $p_{\mathrm{S}}$ is pressure at the southern end of the section (bottom pressure on the Antarctic continental slope; red line in Fig. 1), and $p_{\mathrm{N}}$ is pressure at the same depth, at the northern end of the section (green line in Fig. 1). Remembering that $f$ is negative in the Southern Hemisphere, this means that an increase in eastward transport requires a drop in Antarctic bottom pressure, or a rise in pressure to the north. If we consider a change involving currents limited to the Southern Ocean, then an increase in transport requires either a change

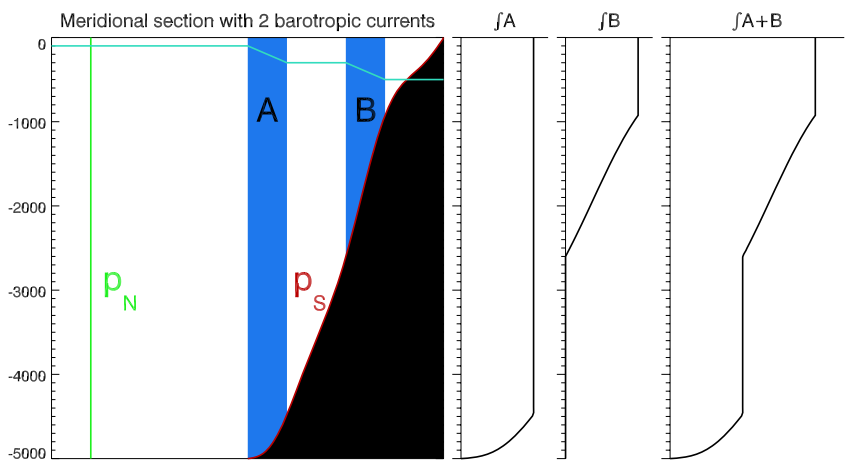

Fig. 1. Schematic showing how two uniform, depth-independent eastward flows (shown as blue-shaded regions $A$ and $B$ ) produce a surface-intensified flow when integrated meridionally across the currents. The left-hand panel represents a meridional section, with Antarctica (black) on the right, and the three right-hand panels show the meridional integrals of currents $A, B$, and $A+B$ as a function of depth. Cyan illustrates how pressure varies on any horizontal surface, and the green and red lines show where pressures $p_{\mathrm{N}}$ and $p_{\mathrm{S}}$ are measured.

in stratification which extends over the entire world ocean north of the Southern Ocean, a similarly distributed bottom pressure change, or a bottom pressure change on the Antarctic continental slope.

The scenario involving a change in the stratification of the world ocean was considered by Allison et al. (2011) using a model based on the simple 1.5-layer model of Gnanadesikan and Hallberg (2000), and it was shown that the appropriate adjustment timescale for reasonable mixing coefficients and wind stress is measured in hundreds of years. Thus, we should be able to exclude this option from consideration for the multidecadal and shorter timescales considered here.

The alternative scenario requires bottom pressure changes to north and/or south. Since bottom pressure is a measure of the column-integrated mass of the ocean, a fall in pressure in one region must be accompanied by a rise elsewhere in order to conserve mass. If the fall is in the Southern Ocean (and perhaps focused on the Antarctic continental slope), and the rise is in the region to the north, then mass conservation and consideration of the relative areas occupied by these regions shows that a large pressure drop to the south would be accompanied by only a small rise to the north. In other words, the pressure change associated with a change in transport will be seen to the south of the current, with only a small compensating rise elsewhere. This is what is seen in barotropic models (Hughes and Stepanov, 2004), with the slight complication that a link with form stress in the Drake Passage region means the rise tends to be focused in the Pacific. The generality of the argument, though, suggests that it ought to hold for variability on all timescales shorter than hundreds of years. 
Given this scenario, we should expect transport to be associated with a bottom pressure change to the south, so that for anomalies from the time mean (indicated by a prime, e.g. $\left.T^{\prime}\right)$ we can ignore the northern pressure and write Eq. (1) as

$T^{\prime}(z)=\frac{p_{\mathrm{S}}^{\prime}(z)}{\rho f}$,

and the depth-integrated transport anomaly $\psi^{\prime}=$ $\int_{-H}^{0} T^{\prime}(z) \mathrm{d} z$ would be given by $1 /(\rho f)$ times the depthintegrated pressure anomaly on the southern boundary, or $H /(\rho f)$ times the depth-averaged pressure anomaly. Here, $H$ is the depth range covered by the region of the Antarctic continental slope over which this average is taken. Hence $\psi^{\prime} / p_{\mathrm{av}}^{\prime}=H /(\rho f)$, where $p_{\mathrm{av}}^{\prime}$ is the depth-averaged southern boundary pressure. To be truly general, we should also note that this assumes that the Coriolis parameter, $f$, averaged over the latitudinal distribution of the current is constant in time. Changes in the path of the current would break this relationship, but we find no evidence for the importance of this effect in what follows.

Assuming that the slope covers a $5 \mathrm{~km}$ depth range and the relevant current is at $65^{\circ} \mathrm{S}$, we obtain

$\psi^{\prime} / p_{\mathrm{av}}^{\prime}=H /(\rho f)=-3.69 \mathrm{~Sv} \mathrm{mbar}^{-1}$.

Being based purely on geostrophic balance, this relationship should hold on all timescales longer than a few days and shorter than hundreds of years, even as the physical processes involved in producing transport variations change. What is not so clear is whether $p_{\mathrm{S}}^{\prime}=p_{\mathrm{av}}^{\prime}$ at all depths, or whether the boundary pressure will vary as a function of depth. The ACC itself is stronger at the surface than at depth, so on sufficiently long timescales we would expect variations in the flow to be surface-intensified, and this would mean that the associated $p_{\mathrm{S}}^{\prime}$ would be larger than $p_{\mathrm{av}}^{\prime}$ in shallow water, and smaller in deep water. This could be due to baroclinic variability involving density changes over the continental slope.

It is, however, possible to produce a similar surface intensification of the pressure signal (and hence of the meridionally integrated flow) with no density change over the slope, if there are geostrophic currents on the continental slope. In simple terms (see Fig. 1), if a barotropic current flows along the deeper part of the slope, then it contributes to the meridional-integral transport at all depths, but a flow on the shallower part of the slope only contributes to the shallower integral, thus representing a surface intensification of the meridionally integrated current, albeit with no surface intensification of the current at any particular horizontal position.

Turning now to the surface signal, any barotropic variability will affect sea level in the same way as bottom pressure. When density can change though, unlike for bottom pressure, there is no longer any direct relationship between sea level and integrated transport. Rather, we would expect a surfaceintensified current to be reflected in a sea level change which is larger than the associated average pressure anomaly.
In summary, we expect a $1 \mathrm{~Sv}$ eastward transport anomaly to be associated with a $3.69 \mathrm{mbar}$ fall in pressure averaged as a function of depth over $5 \mathrm{~km}$ of Antarctic continental slope. Averaging inverse barometer-corrected sea level (or sub-surface pressure) in the same way will produce the same relationship with transport if the variations are barotropic, but at longer timescales density changes are likely to decouple sea level from bottom pressure. If this occurs as the transport becomes surface intensified, then we would expect the sea level signal to become larger than the bottom pressure signal at long timescales.

\section{Model diagnostics}

We investigate the southern mode in two model runs. Both are forced by realistic winds and fluxes and use the NEMO model infrastructure and the ORCA model, on a grid which is regular in longitude south of about $20^{\circ} \mathrm{N}$, with latitude spacing chosen to produce near-square grid cells. North of $20^{\circ} \mathrm{N}$, the grid distorts in a manner which produces a seam in the Arctic, avoiding any singularity at the pole (two singularities in the grid mesh both occur over land). The first run is a 50year run at quarter degree resolution, covering 1958-2007, and the second is a 20 -year run at $1 / 12^{\circ}$ resolution, covering 1978-2007. More details of these model runs are given in Blaker et al. (2013), where they are referred to as N206 and ORCA12. The 50-year run is our main tool, but we will use the higher resolution run to test the robustness of our results. All diagnostics are based on 5-day average fields.

In order to remove erroneous effects of the model's Boussinesq approximation, and also to avoid any complications due to sources and sinks of volume (from rivers, evaporation and precipitation), the global-average bottom pressure is calculated for each 5-day average, and this is subtracted from the value at each position, with the corresponding correction also being made to sea level. No atmospheric pressure forcing is applied in these models, so bottom pressures after these corrections are all associated with ocean dynamics, and sea level is related to ocean dynamics plus the mean density change of the ocean. Correlations and variance explained will all be based on time series after removal by least-squares fitting of a linear trend, annual and semiannual cycle. In addition, the first 5 years of the 1/4 degree model are discarded for any calculations involving Fourier analysis, to reduce the influence of an initial transient signal.

Figure 2 shows the resulting correlation between bottom pressure and flow through Drake Passage for the two models. It also shows the $H / f$ contours which correspond at $65^{\circ} \mathrm{S}$ to depths of 1,3 and $4 \mathrm{~km}$. The correlations show the expected general form of the southern mode, with strong negative correlations to the south and only weak positive correlations further north. The strongest correlations, typically -0.85 , occur on the continental slope between about 1 and $3 \mathrm{~km}$ depths. 

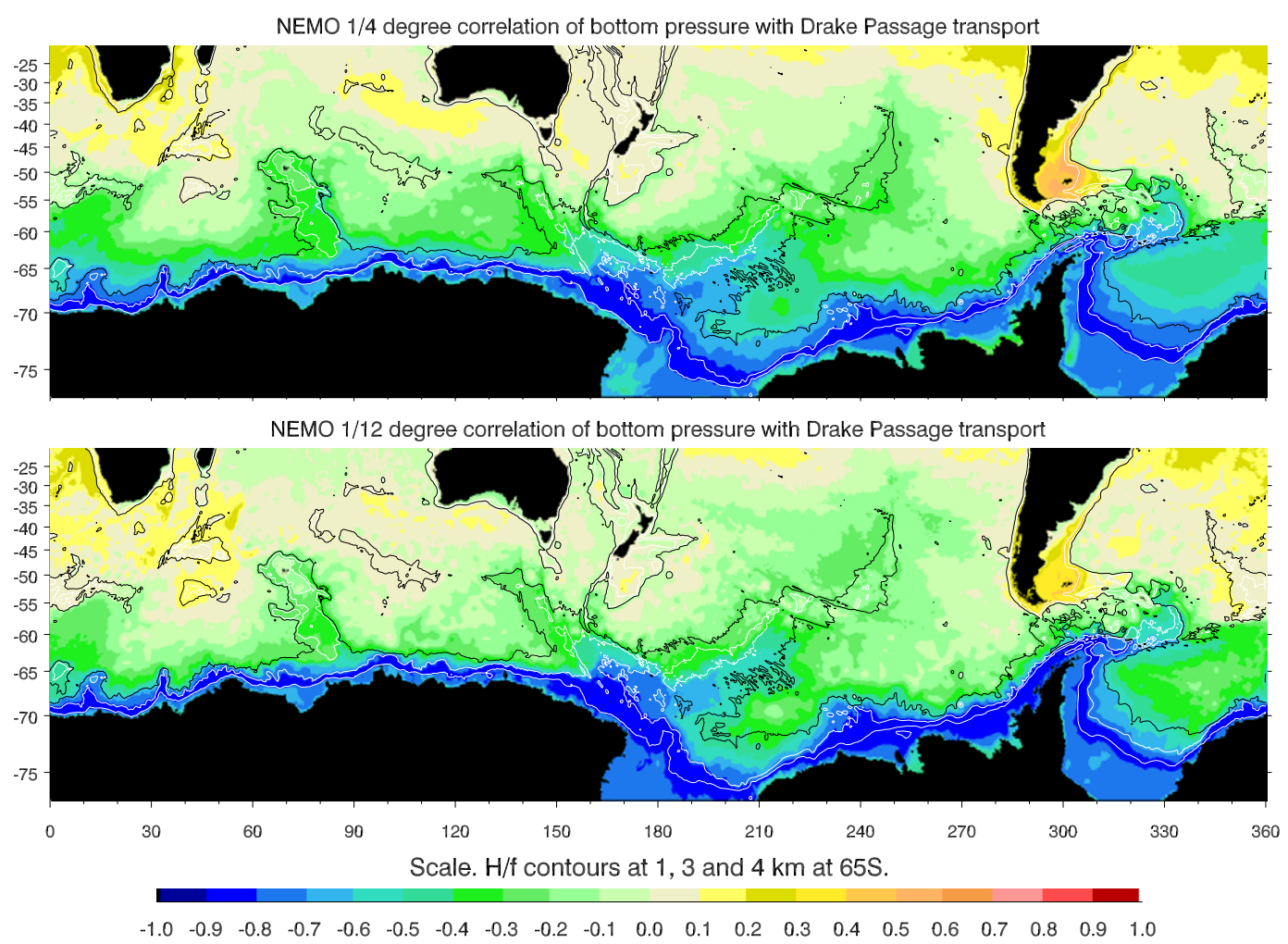

Fig. 2. Correlation between bottom pressure at each point and total flow through Drake Passage, based on (top) 50 years of 5-day means in a $1 / 4^{\circ}$ resolution model, and (bottom) 20 years of 5 -day means in a $1 / 12^{\circ}$ resolution model. All time series have had annual and semiannual cycles and trend removed beforehand. Contours highlight the $H / f$ contours which, at $65^{\circ} \mathrm{S}$, correspond to depths of $1 \mathrm{~km}, 3 \mathrm{~km}($ white) and $4 \mathrm{~km}$ (black).

To produce a time series of depth-averaged continental slope pressure, we define the Antarctic continental slope as the area in Fig. 2 for which correlations are less than (more strongly negative than) -0.5 . We divide this area into 50 regions each representing a $100 \mathrm{~m}$ depth range, between 0 and $5000 \mathrm{~m}$. We area-average the bottom pressures in each of these regions to produce 50 bottom pressure time series, and then average the 50 time series to produce a depthaveraged time series. This is scaled by our predicted factor of $-3.69 \mathrm{~Sv}$ mbar $^{-1}$ and plotted in Fig. 3, together with the transport time series (with trend, annual and semiannual cycles not removed). The time series match well at all timescales. Correlations (after removing trend, annual and semiannual) are 0.92 , rising to 0.98 for a running annual average. Furthermore, the amplitude is correct, so that the scaled pressure explains $85 \%$ and $96 \%$ of the transport variance, for the unsmoothed and smoothed cases respectively, which is as high as is possible with these correlation coefficients (the square of the correlation). Southern boundary pressure is a very good measure of transport, in precisely the way predicted by the kinematic argument.

The lower panel of Fig. 3 shows the same pressure time series (this time converted to an equivalent sea level), together with the corresponding average sea level time series, and their difference, which represents the steric sea level signal, resulting from density changes above the continental slope. It is apparent that, at the longest timescales, sea level is dominated by steric variability, but at shorter timescales sea level and bottom pressure are equivalent. The steric signal shows annual and semiannual variability, but little else except at the longest periods.

More insight into timescales comes from the spectra and cross-spectral squared coherence, phase and gain, between these bottom pressure, sea level, and scaled transport (divided by $-3.69 \mathrm{~Sv} \mathrm{mbar}^{-1}$ ) time series, as shown in Fig. 4 . The spectra look almost identical except at the longest timescales, with the steric signal becoming stronger than the bottom pressure signal at 10 -year period. The squared coherences (when the phase lag is zero or $180^{\circ}$, this is analogous to a squared correlation coefficient, as a function of frequency) show that, although sea level becomes decoupled from bottom pressure at the long periods, it remains coherent with transport (although slightly less coherent than pressure). After applying our predicted (negative) correction factor, the phase lags are close to zero. In this case the gain can be thought of as a linear regression coefficient as a function of frequency (we perform the regression both ways round to obtain two estimates of the ratio between the pairs of 

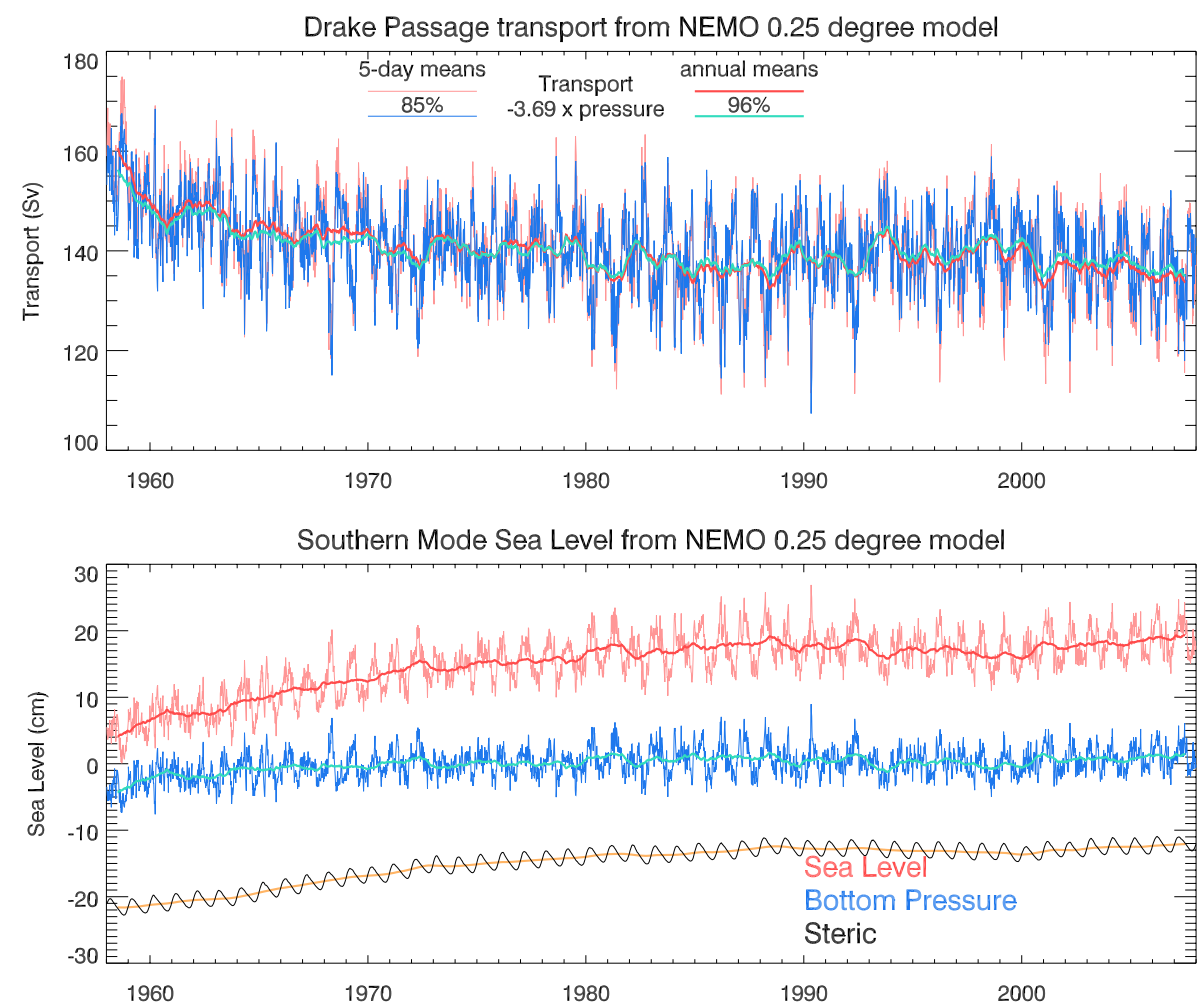

Fig. 3. Time series from the $1 / 4^{\circ}$ model. Top: flow through Drake Passage before (pink) and after (red) applying a running 12-month smoother, and $-3.69 \mathrm{~Sv}_{\text {mbar }}{ }^{-1} \times$ depth-averaged southern boundary pressure anomaly (blue and cyan), with a vertical offset applied to match. Numbers give the percentage of transport variance explained by the corresponding scaled pressure time series. Bottom: the pressure time series used to plot the upper panel (blue and cyan), converted to equivalent sea level units, together with the analogous sea level time series (pink and red), and the difference sea level - bottom pressure (black and orange). Note that the curves are shown with trend, annual, and semiannual cycles included, although these are removed when calculating correlations.

quantities plotted). The gains show that bottom pressure has the same relationship with transport at all timescales, but sea level shows a bigger amplification at longer timescales. Apart from the annual and semiannual periods, sea level and bottom pressure are almost the same at periods of 5 years and shorter, but sea level starts to become amplified between 5year and 10 -year periods.

This is as expected for a transport mode which becomes surface-intensified at periods longer than a few years. But for such a mode, although the relationship between transport and depth-averaged boundary pressure is independent of frequency, the relationship between transport and boundary pressure at any particular depth is frequency-dependent, just as for sea level (after all, bottom pressure and sea level become indistinguishable in sufficiently shallow water). So how is it that we see such strong correlations on the continental slope in Fig. 2?

We investigate this by fitting boundary pressure at each depth on transport, with a range of bandpass filters applied (Fig. 5). We perform the regression both ways round, in each case plotting the resulting ratio $p^{\prime} / \psi^{\prime}$, with for comparison the predicted average value $-1 / 3.69=-0.271 \mathrm{mbar} \mathrm{Sv}^{-1}$ in black. If there is noise in both $p^{\prime}$ and $\psi^{\prime}$, then we would expect the upper panels to underestimate the true magnitude of the fitted value, and the lower panel to overestimate, thus giving an idea of the uncertainty in the fit.

The form of the curves varies with frequency, from almost constant with depth at high frequency to highly surface intensified at low frequency. This represents the form of the meridionally integrated current anomaly as a function of depth, displaying the expected tendency to surface intensification at long period. It shows that, when comparing local pressure to depth-integrated transport, the ratio is frequencydependent in shallow water and at great depth, but there is a mid-depth region around $2 \mathrm{~km}$ where the ratio is almost independent of frequency. This explains the presence of very high correlations on the continental slope seen in Fig. 2. The dropoff of correlations in deeper and shallower water is partly due to this effect, and partly to increased noise in shallower and deeper water. Noise tends to be least at mid-depth because this is where the slope is at its steepest, and steep topography acts to suppress local eddy variability and enhance rapid communication of pressure signals by continental shelf waves. 

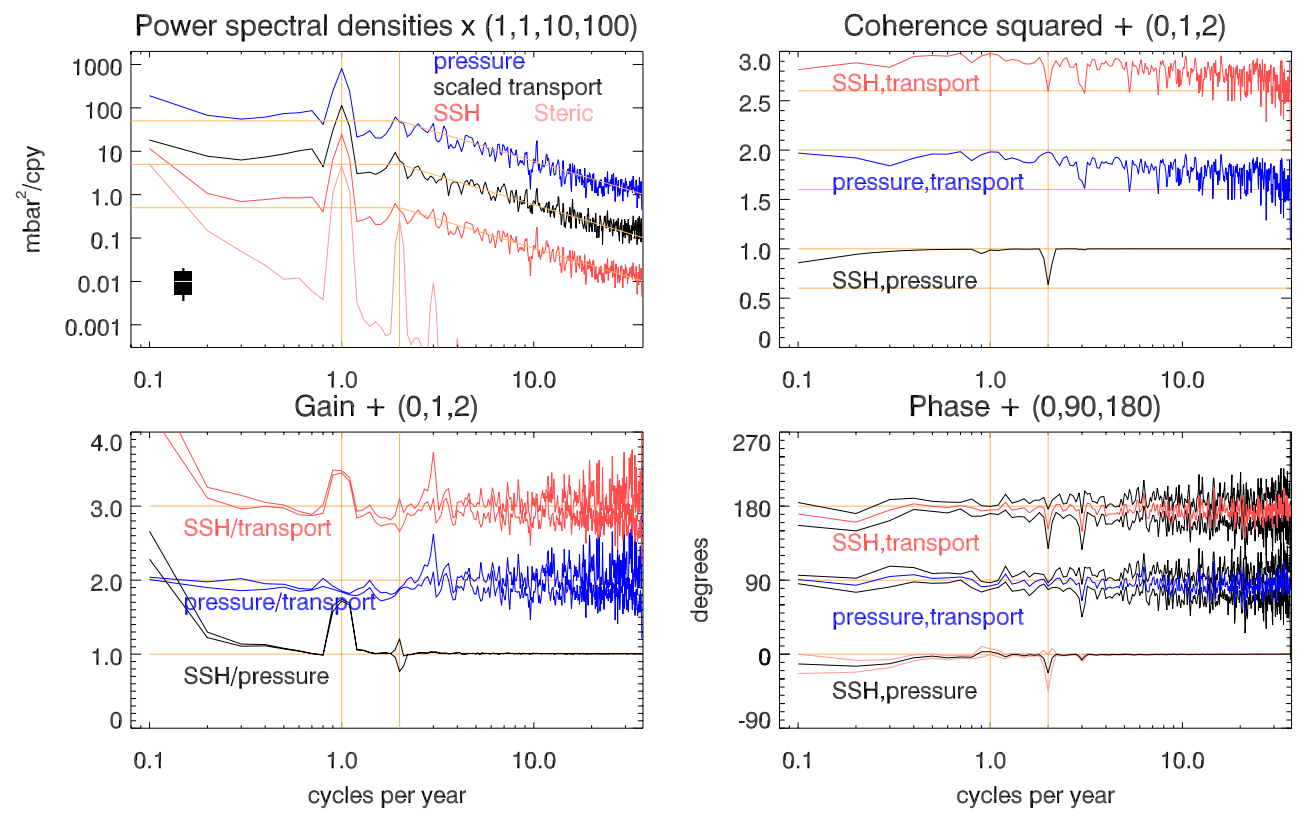

Fig. 4. Spectra and cross-spectral analyses based on time series from the $1 / 4^{\circ}$ model. Top left: power spectra for depth-averaged southern boundary pressure (blue), the analogous sea level (sub-surface pressure) time series (red), the difference sub-surface minus bottom pressure (pink), and Drake Passage transport divided by $-3.69 \mathrm{~Sv} \mathrm{mbar}^{-1}$. Other panels show the squared coherence, gain, and phase lag between pairs of these time series as described in the legends. Orange lines are for guidance and show the offsets applied to the different curves, as well as the annual and semiannual frequencies and, in the case of squared coherence, the value representing significance at the $95 \%$ level. The phase plots include $2 \sigma$ error bounds, and the power spectra plot includes a scale bar for the uncertainty (95\% and $99 \%)$ to be applied at each individual frequency (band averages have smaller errors). Two versions of the gain are shown, representing ratios $a / b$ derived from regressions of $a$ on $b$, and from $b$ on $a$, where $a$ and $b$ are the two time series. When both time series contain noise, the true relationship between them tends to lie between these two estimates.

As noted in the section on kinematics, it is possible for the meridionally integrated flow to be surface-intensified even in the case of a purely barotropic current, if part of the current flows on the continental slope. We can investigate this by calculating the geostrophic current associated with the observed pressure signal, multiplying by depth, and integrating from shallow water out to a chosen isobath. If a significant part of the total transport anomaly results from flows on the continental slope, then the total transport minus this diagnosed slope transport should have smaller variance than the total transport.

In Fig. 6, we show the percentage of total transport variance explained by such slope currents, after integrating out to different depth contours. We split the variance into high frequency (periods shorter than 2 years) medium (periods 2 6 years) and low frequency (periods longer than 6 years). We also consider three versions of the assumed depth-averaged geostrophic current, based on the bottom pressure, sea level, and the average of the two (all three are the same in the case of barotropic variability, which is almost exactly the case at high frequency).

The figure shows that the percentage of transport which is accounted for by flows on the continental slope increases as period increases. In the quarter-degree model, about $30 \%$ of variance in the 2-6-year band is accounted for by flows in the region shallower than $3.2 \mathrm{~km}$, and more than half the longer period variance is accounted for by flows in the region shallower than $2 \mathrm{~km}$. The similarity between results based on sea level and on bottom pressure suggests that the relevant flow is predominantly barotropic. The $1 / 12$ degree model shows even higher percentages at periods up to 6 years, but the increase at longer periods is less dramatic than in the $1 / 4$ degree case, perhaps because of the shorter time series available. This tells us that the surface intensification of the meridionally integrated flow is at least partly a result of an increased tendency for barotropic currents to flow higher up the continental slope at lower frequency. Such flows are enhanced in the higher resolution model, which is better able to represent them.

These circumpolar-average pressure and sea level fields only represent a proxy for the transport. In order to look in more detail at the regions over which transport is correlated with the flow through Drake Passage, we have also calculated depth-integrated and meridionally integrated zonal transports, integrated over sections reaching northwards from Antarctica to each grid point. Figure 7 shows how the resulting time series, filtered over the same three bands as in Fig. 6, relate to the similarly filtered Drake Passage transport. In this 

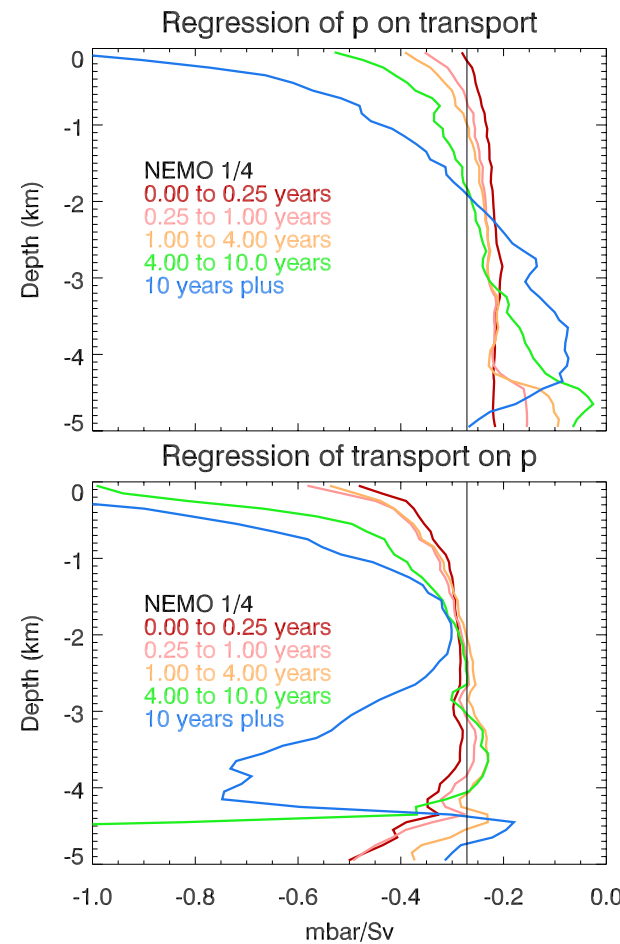

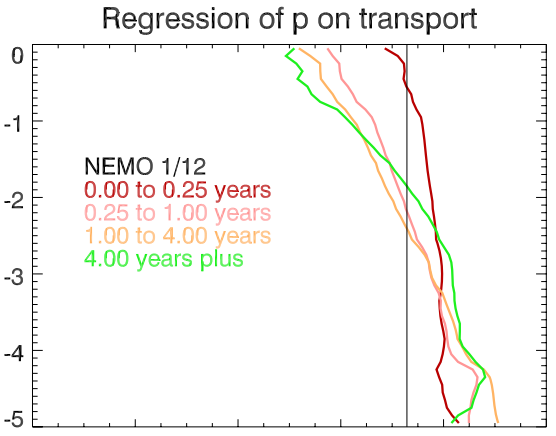

Regression of transport on $p$

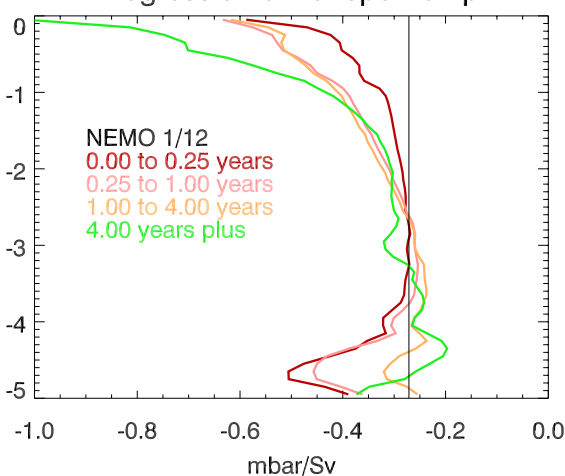

Fig. 5. Ratio of pressure $p$ over transport $\psi$ derived from linear regression of one on the other after applying various bandpass filters given in the legends. Upper panels show $a$ from the regression $p=a \psi+b+$ noise, and lower panels show $1 / A$ from the regression $\psi=A p+B+$ noise. When both time series contain noise, the true relationship tends to lie between these two estimates. Left-hand panels are for the $1 / 4^{\circ}$ model and right-hand panels for the $1 / 12^{\circ}$ model. The black line shows the kinematic estimate for the depth-averaged coefficient: $\left(-3.69 \mathrm{~Sv} \mathrm{mbar}^{-1}\right)^{-1}$
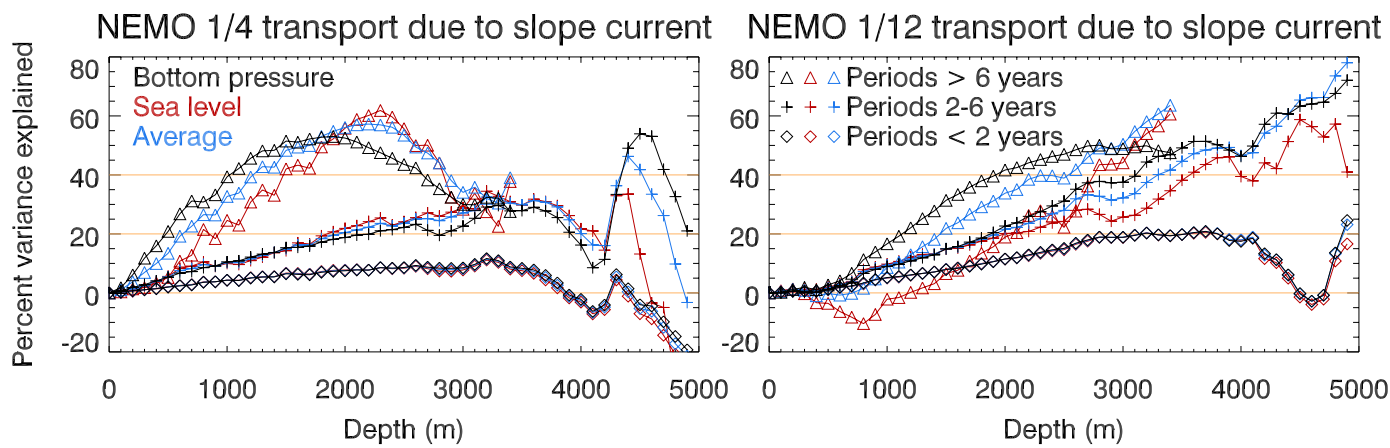

Fig. 6. Estimate of the percentage of total transport variance which is accounted for by flows on the continental slope, plotted as a function of the outermost depth contour to which the continental slope flow is integrated. The calculation is made for periods shorter than 2 years (diamonds), periods 2-6 years (pluses), and periods longer than 6 years (triangles). The currents are estimated by assuming they are barotropic flows associated with the circum-Antarctic average bottom pressure (black), sea level (red) or the average of the two (blue) along each depth contour. Left panel shows results from the $1 / 4^{\circ}$ model, and right panel the $1 / 12^{\circ}$ model.

figure a value of $40 \%$ (say) at one grid point indicates that $40 \%$ of the variance in Drake Passage transport is explained by the flow across a meridional section connecting that grid point to Antarctica.

This analysis supports the conclusions based on Fig. 6: at longer periods, higher percentages are accounted for by flows on the continental slope. There is some geographic variability, with higher values in the Atlantic Ocean and Indian Ocean sectors than in the Pacific sector. Once off the continental slope, the percentage of variance explained rapidly drops, becoming large and negative (white) in the body of the ACC. Presumably some part of the long period transport variability is accounted for by flows in the body of the ACC, but the presence of strong, slowly meandering 


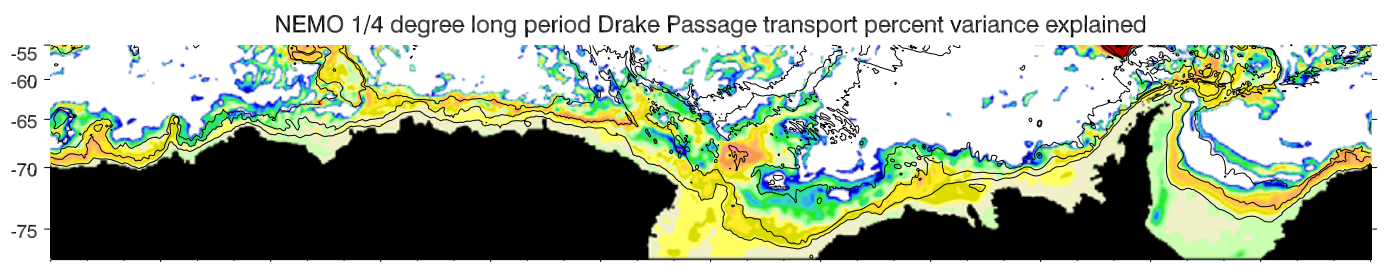

NEMO 1/4 degree medium period Drake Passage transport percent variance explained
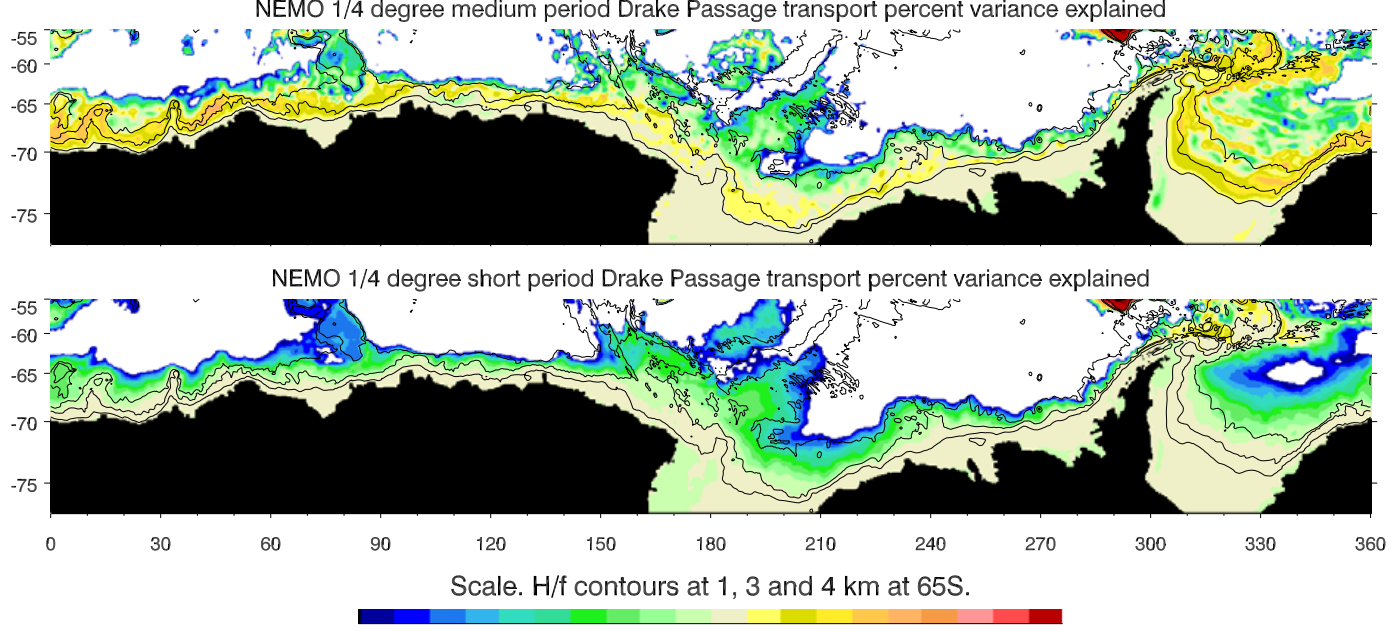

$-100-90-80-70-60-50-40-30-20-100 \quad 102030405060708090100$

Fig. 7. The percentage of variance in Drake Passage transport which is explained by the total zonal transport integrated across a section reaching northwards from Antarctica to each grid point. Annual, semiannual, and linear trend have been subtracted before filtering to pass periods shorter than 2 years (short), periods 2-6 years (medium) and periods longer than 6 years (long) in the three panels. Values below $-100 \%$ are left white.

jets makes it impossible to identify where this component of the transport flows in a meaningful way. It is worth remarking that, after detrending, these long period transport variations are rather small. Standard deviations are 6.26, 1.75 and $1.55 \mathrm{~Sv}$ in the short, medium and long period bands respectively.

\section{Different sections and northern boundaries}

Up to now, we have been considering the circumpolar transport to be defined by the flow through Drake Passage. Also, we have not sought any northern pressure contribution to the transport variability. At one level, this is justified by the success of the southern mode in explaining the transport variability, but it is still worthwhile to look at the questions in more detail.

There are three sections of the Southern Ocean where it is possible to be unambiguous about the integrated transport: south of Africa, Australia, and South America (Drake Passage). The transports through these three sections need not all be the same. They can differ because of recirculations, such as the Indonesian throughflow connecting the Indian and $\mathrm{Pa}-$ cific oceans, and the flow through Bering Strait, connecting the Pacific and Atlantic via the Arctic Ocean. They can also differ if water is accumulating in the various ocean basins, associated with a sea level rise (in the Boussinesq approximation), or if there are net volume sources or sinks from evaporation, precipitation, and river inflow.

We would expect most of these sources of decoupling to be small, especially at long periods, but the Indonesian throughflow is about 13-15 Sv, with significant variability (Gordon et al., 2010), meaning that it could induce significant recirculations around Australia, decoupling the transport variations through the Australian section from those in the other two sections.

We test this by plotting, in Fig. 8, the spectrum of transport variations through Drake Passage and the spectra of differences in transport between the Drake Passage and African sections, and between Africa and Australia. We can see that the difference between flows through Drake Passage and the African section is much smaller than Drake Passage transport variations on all periods longer than about 20 days, and the difference becomes less important at longer periods. However, the difference between the Australian and African sections is important at all periods, and even as large as the total variability in Drake Passage transport in a frequency band between about 6 and 10 cycles per year (periods about 36 to 60 days). Thus, for periods longer than about 20 days it makes sense to think of a single circumpolar transport, plus 

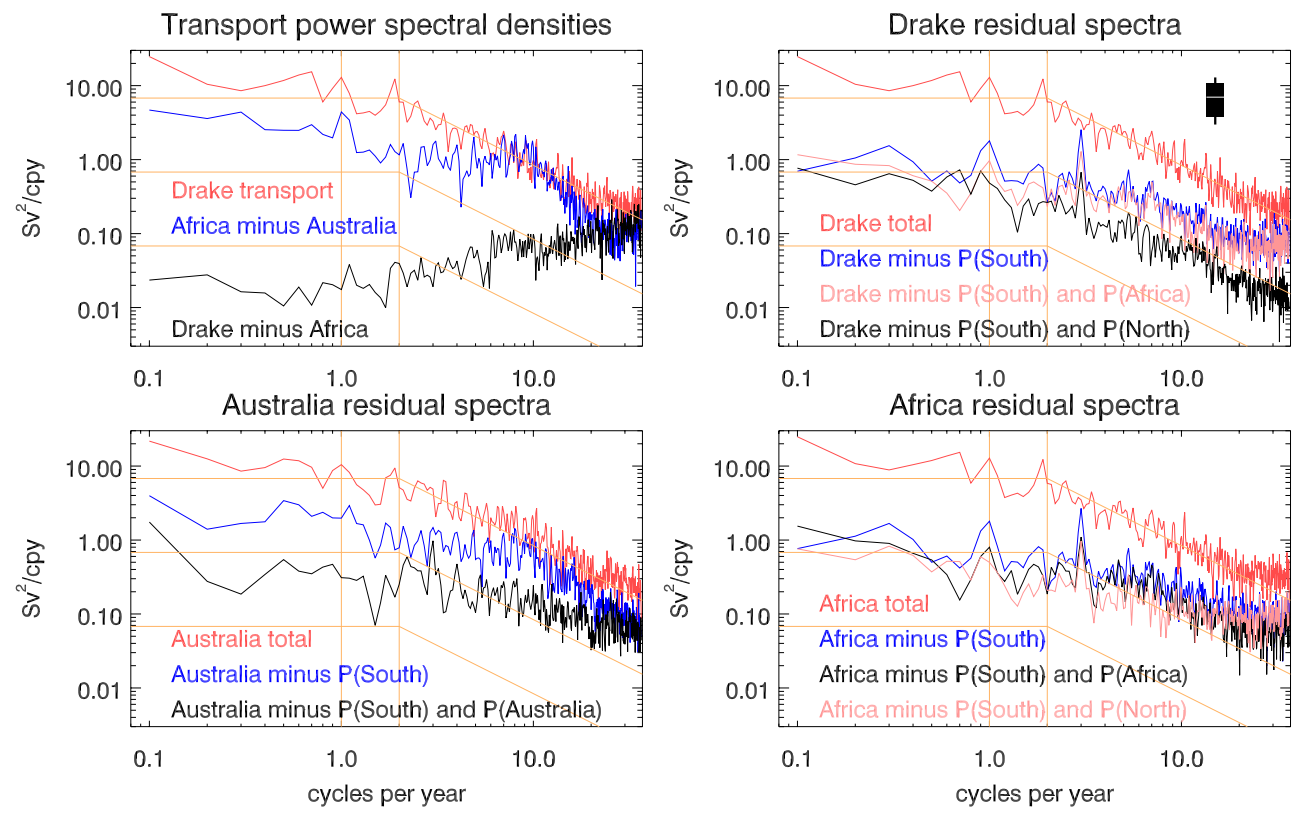

Fig. 8. Power spectra associated with variations in transport through Drake Passage, south of Africa, and south of Australia, from the $1 / 4^{\circ}$ model. The top left panel shows the spectrum of Drake Passage transport (red), that of the difference between Drake Passage transport and transport south of Africa (black), and that of the difference between transports south of Africa and Australia (blue). Other panels show the spectrum of transport through each section (red), of the residual after subtracting the transport accounted for by southern boundary pressure (blue) and of the residual after subtracting the transport accounted for by southern boundary pressure and the corresponding northern boundary pressure (black). Pink curves are like the black curves but use a northern boundary pressure from a different section. Orange lines are for guidance only and show representative spectra separated by factors of 10, together with the annual and semiannual frequencies. The Drake residual plot includes a scale bar for the uncertainty (95\% and $99 \%$ ) to be applied at each individual frequency (band averages have smaller errors).

a second transport recirculating around Australia and closing via the Indonesian throughflow.

These three sections also represent regions where it is possible to clearly define a northern boundary pressure variation, to see whether this plays a significant role in determining the transport through the sections. To do this, we form three new pressure time series in a manner analogous to the formation of the southern mode $p_{\text {av }}$ time series. We define a longitudelatitude box including the southern boundaries of the three continents, and isolate the regions which are unambiguously on the continental slope (i.e. not connected to topography in the wider Southern Ocean). We area-average the bottom pressures in each depth bin, then form a depth average over each of the three continental slopes. Increased, poorly correlated variability in deep regions leads us to confine the depth average to depths shallower than $4500 \mathrm{~m}$ in Drake Passage, $4000 \mathrm{~m}$ south of Africa and $3600 \mathrm{~m}$ south of Australia. This gives us three new time series: $\mathrm{P}$ (North) in Drake Passage, $\mathrm{P}$ (Africa) south of Africa and P(Australia) south of Australia, in addition to the southern mode pressure averaged to $4500 \mathrm{~m}$ which we now refer to as $\mathrm{P}($ South). We then plot the transport spectra for each section, the residual spectrum after subtracting the best fit on $\mathrm{P}$ (South), and the residual after subtracting the best simultaneous fit on $\mathrm{P}$ (South) and the appropriate northern boundary pressure.
The extra information from the northern boundary has the greatest influence in the case of the Australian section. Here, $\mathrm{P}($ South) alone explains $69.2 \%$ of the variance, increasing to $90.4 \%$ when using $\mathrm{P}($ South) and $\mathrm{P}($ Australia), with $\mathrm{P}($ Australia) playing an important role at all frequencies, and especially in the period range 36-60 days.

In the case of Drake Passage, the northern pressure also adds useful information at almost all frequencies, but the impact is greatest at periods shorter than about 6 months. Variance explained increases from $84.7 \%$ using just $\mathrm{P}$ (South) to $94.7 \%$ when using $\mathrm{P}$ (South) and $\mathrm{P}$ (North).

The case of the African section is more equivocal, with $84.0 \%$ of transport variance being explained by $\mathrm{P}($ South), increasing slightly to $89.4 \%$ when using $\mathrm{P}$ (South) and $\mathrm{P}$ (Africa). Africa is the most difficult continent to derive a meaningful northern pressure for because of the strong eddy variability associated with the Agulhas current and retroflection, and the complex topography. It is interesting, therefore, to note that we can explain slightly more of the African transport variance $(90.2 \%)$ by fitting on $\mathrm{P}($ South) and $\mathrm{P}($ North) from Drake Passage, with a reduction of variance seen at most periods longer than about 15 days ( 25 cycles per year). This demonstrates that the coherence of transport between Drake Passage and the African section is not all mediated by pressure signals on the southern boundary. There must 
be some route for communication of pressure anomalies between the north of Drake Passage and the African section, to account for the success of $\mathrm{P}$ (North) in accounting for part of the transport south of Africa.

Thus, in this section, we have shown that although the southern mode accounts for most of the transport variability, there is in addition a mode associated with recirculation around Australia, which is reflected in pressure on the Australian continental slope. Northern pressure variations also account for a small fraction of the transport variance in Drake Passage and south of Africa.

It is worth noting that, although the southern mode accounts for $85 \%$ of the Drake Passage transport variance, that is not the same as saying that $85 \%$ of the variance would be accounted for by considering only pressures from the south of Drake Passage and ignoring the north. For one thing, the scaling we have used considers the pressure averaged over $5 \mathrm{~km}$ of continental slope, but there is no route through Drake Passage at $5 \mathrm{~km}$ depth. In fact, as we see in Fig. 2, there is a small positive correlation between transport and pressure to the north of Drake Passage, and we actually find a negative correlation between $\mathrm{P}$ (South) and $\mathrm{P}$ (North), which is necessary if the transport is to squeeze through the reduced depth range without a local increase in the amplitude of the southern boundary pressure. Thus, $\mathrm{P}($ North) plays a significant role in Drake Passage, but adds little extra information to $\mathrm{P}$ (South) because the main role for $\mathrm{P}($ North) comes from a component which is anticorrelated with $\mathrm{P}$ (South).

\section{Conclusions}

We have investigated the relationship between the southern mode and Antarctic circumpolar transport fluctuations in two multidecadal runs of eddy-permitting ocean models. We have found that, interpreted as the continental slope pressure averaged as a function of depth over $5 \mathrm{~km}$ depth range of the Antarctic continental slope, the southern mode is an excellent, direct measure of transport with a simple conversion factor of $-3.69 \mathrm{~Sv} \mathrm{mbar}^{-1}$ applying at all periods from about 20 days to multidecadal. This works well for transport through Drake Passage and south of Africa. South of Australia, an additional source of variability comes from the Indonesian throughflow, and can be seen via its influence on depth-averaged bottom pressure on the Australian continental slope.

Variability is essentially barotropic at periods shorter than about 5 years, but even barotropic variability can produce surface intensification in the meridionally integrated current if part of the current flows in shallow regions of the continental slope, and this process does indeed appear to account for a significant part of the variability at periods longer than 2 years, especially in the finer resolution model.
At periods longer than about 5 years, baroclinic processes become important, decoupling sea level from bottom pressure. As the meridionally integrated flow becomes more surface-intensified, the bottom pressure signal in shallow water becomes larger than the depth average, as does the sea level signal.

When it comes to monitoring changes in ACC transport, this means that we have to consider two cases. For periods shorter than about 5 years, there is little frequency dependence in the size of pressure or sea level responses to transport variations. Large-scale averaged pressures from GRACE satellite gravity, sea levels from altimetry and tide gauges, and direct bottom pressure measurements from in situ instruments, can all contribute to the measurement of the southern mode in a straightforward way. At longer periods, a frequency-dependent gain must be used for each kind of measurement, with the gain depending on the particular spatial averaging appropriate to the measurement used. The only form of measurement which is directly related to the transport is bottom pressure, averaged so as to give equal weighting to equal depth-range intervals or, approximately, bottom pressure averaged over the narrow, steep section of continental slope between about 1 and $3 \mathrm{~km}$ depth. Unfortunately, this is too narrow a strip to be cleanly picked out from GRACE measurements, which cannot resolve such small spatial scales. It seems that a strategy involving frequencydependent gain is unavoidable.

Acknowledgements. This work was funded by NERC, partly as a contribution to NOC National Capability science, and partly by grants NE/H019812/1 and NE/I023384/1. The model simulations analysed in this work made use of the facilities of HECToR, the UK's national high-performance computing service, which is provided by UoE HPCx Ltd at the University of Edinburgh, Cray Inc. and NAG Ltd, and funded by the Office of Science and Technology through EPSRC's and NERC's High End Computing Programme. Thanks to Wilbert Weijer and an anonymous reviewer, whose comments and suggestions have improved this paper.

Edited by: M. Hecht

\section{References}

Allison, L. C., Johnson, H. L., and Marshall, D. P.: Spin-up and adjustment of the Antarctic Circumpolar Current and global pycnocline, J. Mar. Res., 69, 167-189, 2011.

Aoki, S.: Coherent sea level response to the Antarctic Oscillation, Geophys. Res. Lett., 29, 1950, doi:10.1029/2002GL015733, 2002.

Blaker, A. T., Hirschi, J. J.-M., McCarthy, G., Sinha, B., Taws, S., Marsh, R., Coward, A., and de Cuevas, B.: Historical analogues of the recent extreme minima observed in the Atlantic meridional overturning circulation at $26^{\circ} \mathrm{N}$, Clim. Dynam., submitted, 2013.

Cunningham, S. A., Alderson, S. G., King, B. A., and Brandon, M. A.: Transport and variability of the Antarctic Circum- 
polar Current in Drake Passage, J. Geophys. Res., 108, 8084, doi:10.1029/2001JC001147, 2003.

Gnanadesikan, A. and Hallberg, R.: On the relationship of the circumpolar current to Southern Hemisphere winds in coarseresolution ocean models, J. Phys. Oceanogr., 30, 2013-2034, 2000.

Gordon, A. L., Sprintall, J., Van Aken, H. M., Susanto, D., Wijffels, S., Molcard, R., Ffield, A., Pranowo, W., and Wirasantosa, S.: The Indonesian throughflow during 2004-2006 as observed by the INSTANT program, Dyn. Atmos. Ocean., 50, 115$128,2010$.

Hibbert, A., Leach, H., Woodworth, P., Hughes, C. W., and Roussenov, V.: Quasi-biennial modulation of the Southern Ocean Coherent Mode, Q. J. Roy. Meteor. Soc., 136, 755-768, 2010.

Hughes, C. W. and Meredith, M. P.: Coherent sea level fluctuations along the global continental slope, Philos. T. Roy. Soc. Lond. A, 364, 885-901, doi:10.1098/rsta.2006.1744, 2006.

Hughes, C. W. and Stepanov, V. N.: Ocean dynamics associated with rapid $J_{2}$ fluctuations: importance of circumpolar modes and identification of a coherent Arctic mode, J. Geophys. Res., 109 C06002, doi:10.1029/2003JC002176, 2004.

Hughes, C. W., Meredith, M. P., and Heywood, K.: Winddriven transport fluctuations through Drake Passage: a Southern Mode, J. Phys. Oceanogr., 29, 1971-1992, 1999.
Hughes, C. W., Woodworth, P. L., Meredith, M. P., Stepanov, V., Whitworth, T., and Pyne, A.: Coherence of Antarctic sea levels, Southern Hemisphere Annular Mode, and flow through Drake Passage, Geophys. Res. Lett., 30, 1464, doi:10.1029/2003GL017240, 2003.

Kusahara, K. and Ohshima, K. I.: Dynamics of the wind-driven sea level variation around Antarctica, J. Phys. Oceanogr., 39, 658674, doi:10.1175/2008JPO3982.1, 2009.

Olbers, D. and Lettmann, K.: Barotropic and baroclinic processes in the transport variability of the Antarctic Circumpolar Current, Ocean Dynam., 57, 559-578, doi:10.1007/s10236-007-0126-1, 2007.

Vivier, F., Kelly, K. A., and Harismendy, M.: Causes of largescale sea level variations in the Southern Ocean: analyses of sea level and a barotropic model, J. Geophys. Res., 110, C09014, doi:10.1029/2004JC002773, 2005.

Weijer, W. and Gille, S. T.: Adjustment of the Southern Ocean to wind forcing on synoptic timescales, J. Phys. Oceanogr., 35, 2076-2089, doi:10.1175/JPO2801.1, 2005.

Woodworth, P. L., Vassie, J. M., Hughes, C. W., and Meredith, M. P.: A test of TOPEX/POSEIDON's ability to monitor flows through Drake Passage, J. Geophys. Res.-Oceans, 101, 11935-11947, 1996.

Zika, J., Le Sommer, J., Dufour, C., Naveira-Garabato, A., and Blaker, A.: Acceleration of the Antarctic Circumpolar Current by wind stress along the coast of Antarctica, J. Phys. Oceanogr., doi:10.1175/JPO-D-13-091.1, in press, 2013. 